\title{
Failure of the Standard Coupled-Channels Method in Describing the Inelastic Reaction Data: On the Use of a New Shape for the Coupling Potential
}

\author{
S.Erturk $\dagger$, I.Boztosun $\ddagger$ Y.Kucuk $\ddagger$ M.Karakoc $\ddagger$ S.Aydin $\dagger$ \\ $\dagger$ Nigde University, Department of Physics, Nigde-TURKEY. \\ $\ddagger$ Erciyes University, Department of Physics, Kayseri-TURKEY.
}

\begin{abstract}
We present the failure of the standard coupled-channels method in explaining the inelastic scattering together with other observables such as elastic scattering, excitation function and fusion data. We use both microscopic double-folding and phenomenological deep potentials with shallow imaginary components. We argue that the solution of the problems for the inelastic scattering data is not related to the central nuclear potential, but to the coupling potential between excited states. We present that these problems can be addressed in a systematic way by using a different shape for the coupling potential instead of the usual one based on Taylor expansion.
\end{abstract}

PACS numbers: 24.10.-i, 24.10.Eq, 24.10.-v, 24.10.+g

Keywords: Coupled-Channels model, elastic/inelastic scattering, ${ }^{12} \mathrm{C}+{ }^{12} \mathrm{C}$ reaction 


\section{INTRODUCTION}

In this paper, we consider the ${ }^{12} \mathrm{C}+{ }^{12} \mathrm{C}$ reaction as a case study to point out the problems for the inelastic scattering states which have so far remained unsolved, and to address particularly the magnitude problem for the inelastic scattering data. Theoretical calculations using the coupled-channels (CC) method fail to correctly predict the magnitude of the single$2^{+}$and mutual-2 $2^{+}$states data together with the elastic scattering data. In order to get the magnitude right, many futile theoretical attempts have been made for these states. Previous theoretical works show that the shapes of the central real potentials are actually correct, since they explain the elastic scattering data and predict the resonances at the correct energies with reasonable widths. It appears that the failure of the standard methods is mostly related to the inelastic scattering data: The magnitude of the theoretical crosssections is much smaller than the measured experimental data. In this paper, we make further applications of a new coupling potential [1] in describing the scattering observables of the ${ }^{12} \mathrm{C}+{ }^{12} \mathrm{C}$ system.

\section{THE MODEL}

For the spherical nuclei, the nuclear shape and also the shape of the potential between projectile and target nuclei are characterized by a constant radius $R$, which defines the distance of the center of the nucleus from the surface. However, for a deformed nucleus, the radius parameter is no longer constant but depends on the angular location of the point $(R \rightarrow R(\theta, \phi))$. The nucleus ${ }^{12} \mathrm{C}$ we study in this paper is strongly deformed and its collective excitation is taken into account by using the standard deformation procedure based on the Taylor expansion. If the interaction potential between two nuclei is taken to be $U(r-R(\theta, \phi))$, the Taylor expansion about $R=R_{0}$ yields,

$$
U(r-R)=U\left(r-R_{0}\right)-\delta R \frac{\partial}{\partial r} U\left(r-R_{0}\right)+\frac{1}{2 !}(\delta R)^{2} \frac{\partial^{2}}{\partial r^{2}} U\left(r-R_{0}\right)-\ldots
$$

Here, the first term is the usual diagonal optical potential that describes only the elastic scattering and the second and third terms are used to describe the inelastic scattering and to obtain the coupling potentials for the single- $2^{+}$and mutual- $2^{+}$states. $\delta R$ in equation 1 
is given as:

$$
\delta R=R_{i} \sum_{l m} \beta_{l m} Y_{l m}(\theta, \phi)^{*}
$$

with $i$ as the projectile $a$ or the target $A . \beta_{l m}$ is the deformation parameter and it is -0.6 for the ${ }^{12} \mathrm{C}$ nucleus.

In the phenomenological analysis, the real nuclear potential has the square of the WoodsSaxon shape, and the imaginary potential has the Woods-Saxon volume shape. The parameters of the real and imaginary parts are taken from ref. [1].

For the microscopic analysis, the Nucleon-Nucleon double-folding potential [2] is

$$
V_{D F}(R)=\int \rho_{P}\left(r_{1}\right) \rho_{T}\left(r_{1}\right) V_{n n}\left(\left|\vec{R}+\vec{r}_{2}-\vec{r}_{1}\right|\right) d \vec{r}_{1} d \vec{r}_{2}
$$

where $\rho_{P}$ and $\rho_{T}$ are the nuclear matter distributions for projectile and target nuclei respectively, and they are given by

$$
\rho_{P}\left(r_{1}\right)=\rho_{0 P}\left(1+\gamma r_{1}^{2}\right) \exp \left(-\xi r_{1}^{2}\right) \rho_{T}\left(r_{2}\right)=\rho_{0 T}\left[1+\exp \left(\left(r_{2}-c\right) / a\right)\right]
$$

where $\rho_{0 P}=0.1644 \mathrm{fm}^{-3}, \gamma=0.4988 \mathrm{fm}^{-2}$ and $\xi=0.3741 \mathrm{fm}^{-2}$ for projectile and $\rho_{0 T}=0.207$ $\mathrm{fm}^{-3}, \mathrm{c}=2.1545 \mathrm{fm}$, and $\mathrm{a}=0.425 \mathrm{fm}$ for target nuclei.

The M3Y Nucleon-Nucleon effective interaction is taken in the form

$$
V_{n n}(r)=7999 \frac{\exp (-4 r)}{4 r}-2134 \frac{\exp (-2.5 r)}{2.5 r}+J_{00}(E) \delta(s)
$$

where $J_{00}(E)=276\left(1-0.005 \frac{E}{A p}\right)$. The real and imaginary potentials are shown in figure 1 and the parameters are given in table \labelled as DF.

In the new coupled-channels model, we have replaced the usual first derivative coupling potential by a second-derivative coupling potential in Woods-Saxon form which is multiplied by the diffuseness parameter $(a)$ to normalize the units in the calculations. The parameters are given in table $\llbracket$

\section{RESULTS}

We have used both the phenomenological and microscopic potentials to analyze the experimental data of the ${ }^{12} \mathrm{C}+{ }^{12} \mathrm{C}$ reaction at $\mathrm{E}_{\text {Lab }}=74.2 \mathrm{MeV}, 93.8 \mathrm{MeV}$ and $126.7 \mathrm{MeV}$. The experimental data is taken from ref. [3]. The results of our analyzes are displayed in Figure 2 for the ground, 3 for the single- $2^{+}$, and 4 for the mutual- $2^{+}$states in comparison 
with experimental data. Both double-folding and phenomenological potentials provide excellent agreement with the experimental data for the ground state at all energies and a good fit to the single- $2^{+}$state data. However, the mutual $-2^{+}$state prediction is much smaller than the measured one: The standard CC model using double-folding or phenomenological potentials underestimates its magnitude by a factor of 3 to 10 as it has been previously observed [1, 3, 4, 5, 6, 7]. Varying the parameters and changing the shape of the real and imaginary potentials do not provide a complete solution to the problems of this reaction [1]. In order to solve this problem we have used a new coupling potential. This potential has a second-derivative of Woods-Saxon shape and it is compared in figure 1 with the standard coupling potential. This new coupling potential has a repulsive part at short distances and an attractive part at large distances which is related to the orientation of two ${ }^{12} \mathrm{C}$ nuclei at short and large distances [1]. We have been able to obtain excellent agreement with all the available experimental data for the ground, single- $2^{+}$and mutual- $2^{+}$states by using this new coupling potential. The parameters are shown in table \. This new approach solves the magnitude problem of the mutual- $2^{+}$state data, which has been an outstanding problem with this reaction. The results for the ground, single- $2^{+}$and mutual- $2^{+}$states are compared with the standard ones in figures 2, 3 and 4 ,

\section{SUMMARY}

In the present work, we have demonstrated that a consistent solution could be obtained for the problems of the ${ }^{12} \mathrm{C}+{ }^{12} \mathrm{C}$ reaction over a wide energy range. However, we achieve this by using a coupling potential which has a non-standard form. Within the standard formalism, our findings using folding or phenomenological potentials are in agreement with the previous works [1, 3, 4, 5, 6, 7]. Although, within standard approach, these potentials give excellent agreement with the experimental data for the ground state, they are unable to provide a consistent solution to the problems of the inelastic scattering data. This work therefore clearly shows that the standard deformation procedure based on Taylor expansion is inadequate in describing such highly deformed nuclei. It is obvious that the standard formalism should be questioned further. 


\section{Acknowledgments}

This work is supported by the Turkish Science and Research Council (TÜBITAK): Grant No: TBAG-2398 and Erciyes University-Institute of Science: Grant no: FBT-04-15 and FBT-04-16.

[1] I. Boztosun and W.D.M. Rae, Phys. Rev. C 63 (2001) 054607.

[2] M. El-Azab Farid and G. R. Satchler, Nucl. Phys. A438 (1985) 525.

[3] R.G. Stokstad, R.M. Wieland, G.R. Satchler et al., Phys. Rev. C 20 (1979) 655.

[4] P.E. Fry, DPhil Thesis, Oxford University, 1997.

[5] W.D.M. Rae, S.P.G. Chappell and P.E. Fry, Nuovo-Cimento 110A (1997) 1001.

[6] M. Ito, Y. Sakuragi and Y. Hirabayashi, Phys. Rev. C 63, 064303 (2001).

[7] R. Wolf, O. Tanimura, U. Mosel and G.R. Satchler, Z. Phys. 305A (1982) 179. 


\begin{tabular}{lrlllllll}
\hline \hline & $E_{l a b}(\mathrm{MeV})$ & $\mathrm{N}$ & $\mathrm{V}(\mathrm{MeV})$ & $\mathrm{r}_{v}(\mathrm{fm})$ & $\mathrm{a}_{v}(\mathrm{fm})$ & $\mathrm{W}(\mathrm{MeV})$ & $\mathrm{r}_{w}(\mathrm{fm})$ & $\mathrm{a}_{w}(\mathrm{fm})$ \\
\hline $\mathrm{DF}$ & 74.2 & 1.47 & - & - & - & 8.0 & 1.40 & 0.40 \\
& 93.8 & 1.20 & - & - & - & 10.0 & 1.40 & 0.40 \\
& 126.7 & 1.20 & - & - & - & 23.0 & 1.20 & 0.50 \\
\hline \multirow{2}{*}{ New } & 74.2 & - & 285.0 & 0.810 & 1.35 & 6.9 & 1.20 & 0.55 \\
& 93.8 & - & 285.0 & 0.760 & 1.35 & 9.5 & 1.20 & 0.55 \\
& 126.7 & - & 285.0 & 0.800 & 1.35 & 15.0 & 1.20 & 0.55 \\
\hline \hline
\end{tabular}

TABLE I: The parameters of the real and imaginary potentials. For the new coupling potentials, the radius and diffuseness $0.7 \mathrm{fm}$ and the depth is $215.0 \mathrm{MeV}, 230 \mathrm{MeV}$ and $245 \mathrm{MeV}$ for $E_{l a b}=74.2$ $\mathrm{MeV}, 93.8 \mathrm{MeV}$ and $126.7 \mathrm{MeV}$. 


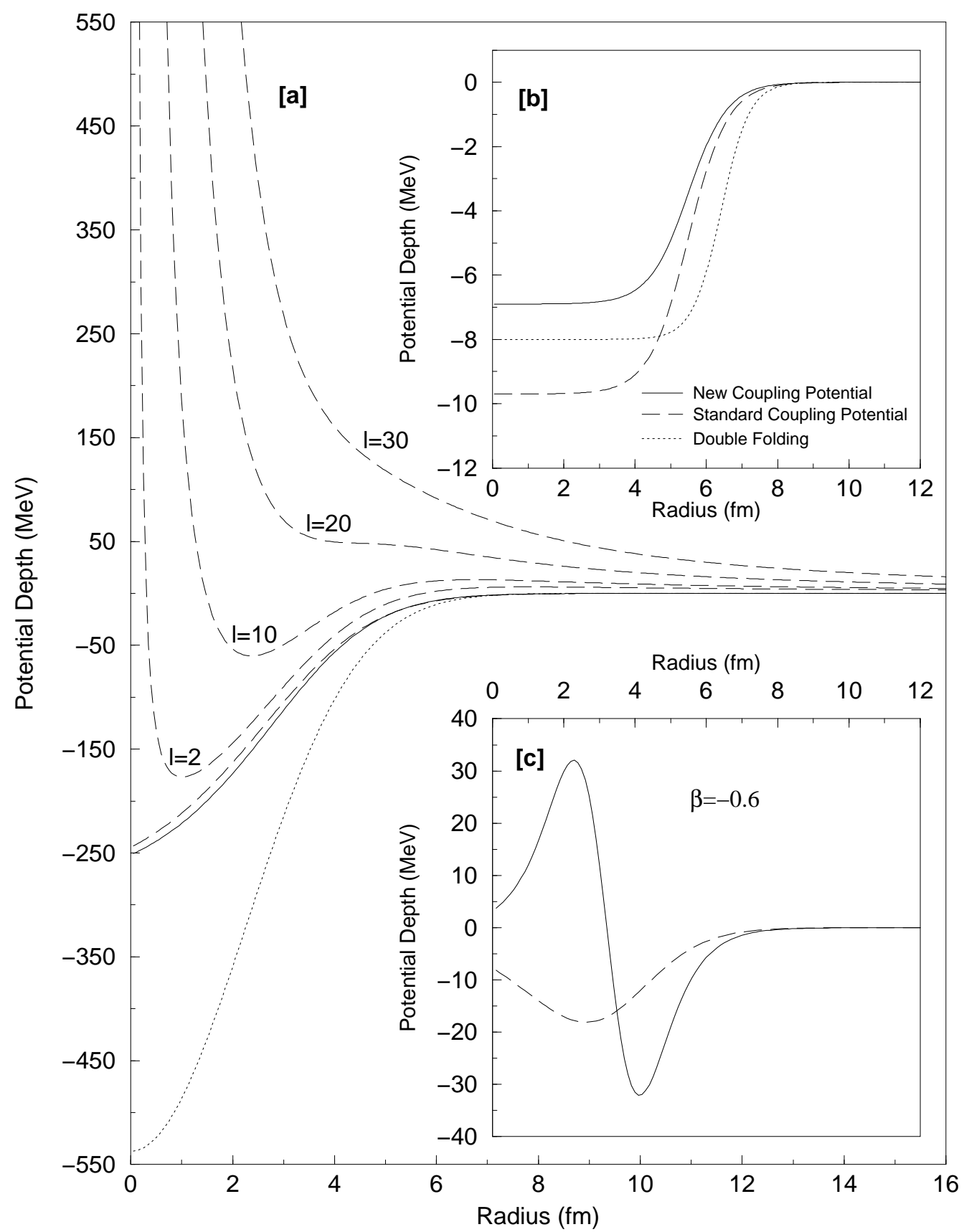

FIG. 1: Potentials: [a] the DF and $\mathrm{WS}^{2},[\mathrm{~b}]$ the imaginary and [c] the standard $\mathrm{WS}^{2}$ and new coupling potentials at $E_{l a b}=74.2 \mathrm{MeV}$. 


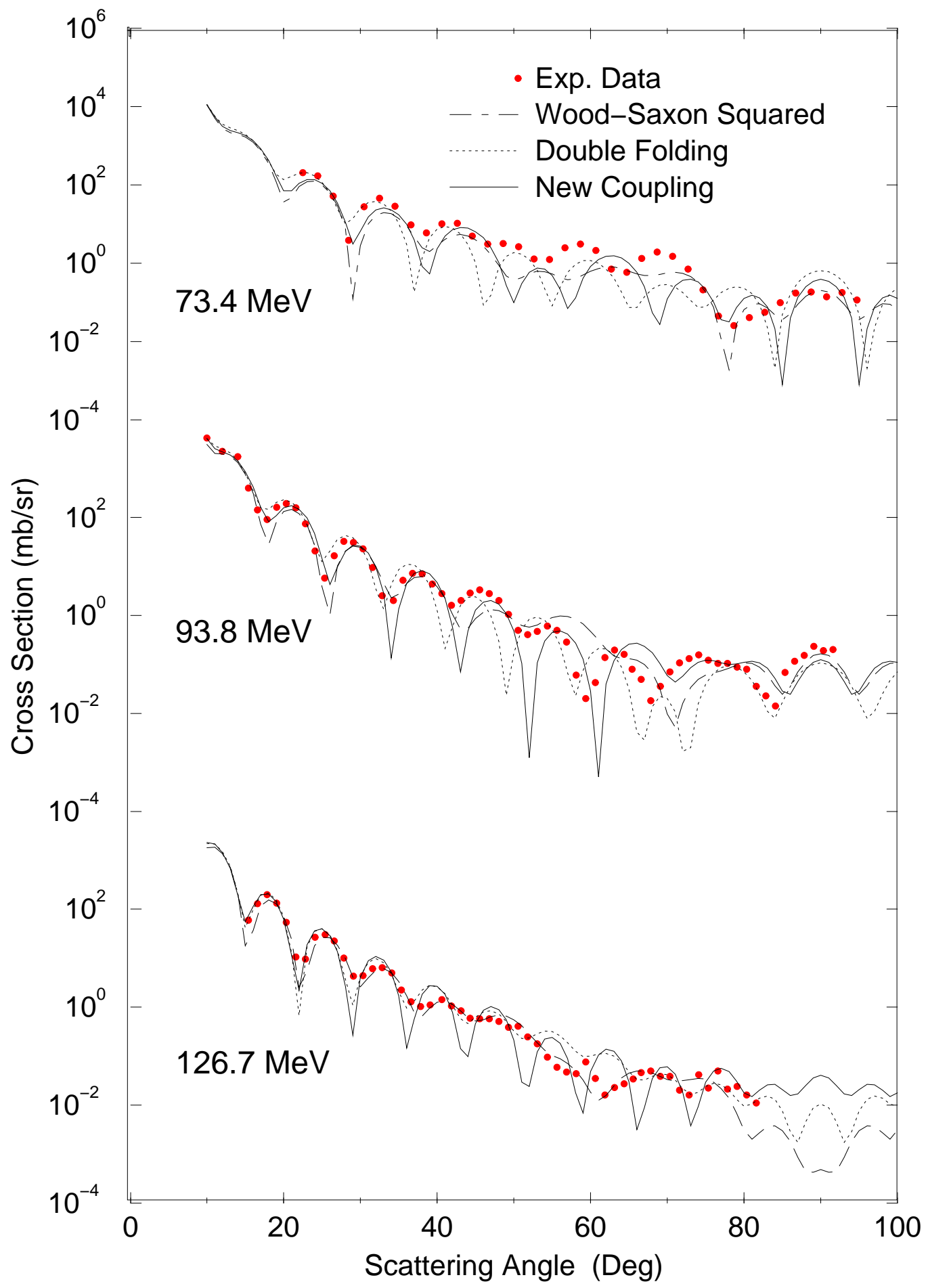

FIG. 2: Ground state results: The dotted and long-dashed lines show the predictions of DF and $\mathrm{WS}^{2}$ potentials with standard coupling while the solid lines show the results of the new coupling potential. 


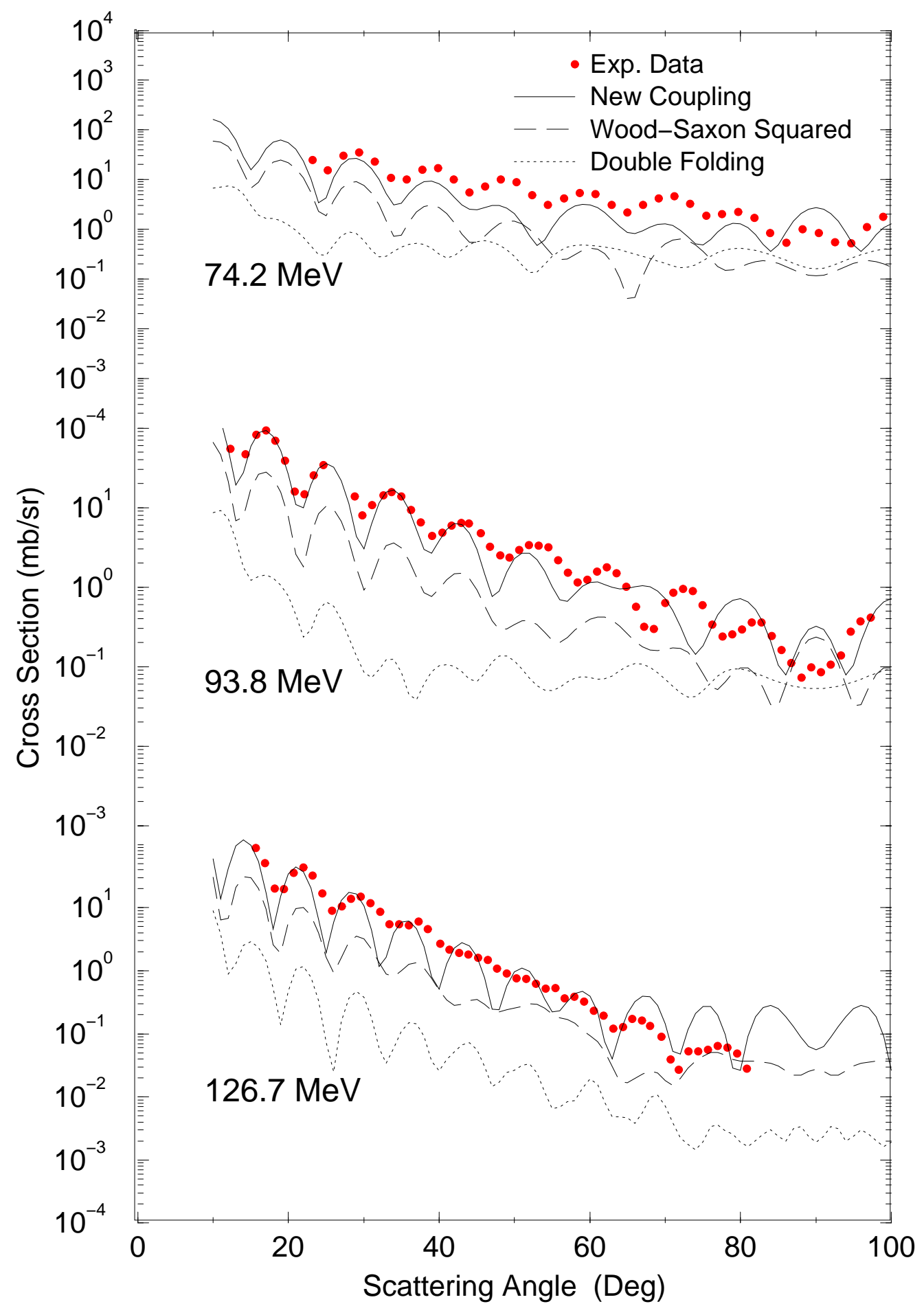

FIG. 3: Single- $2^{+}$state results. The legends are the same as figure 2 


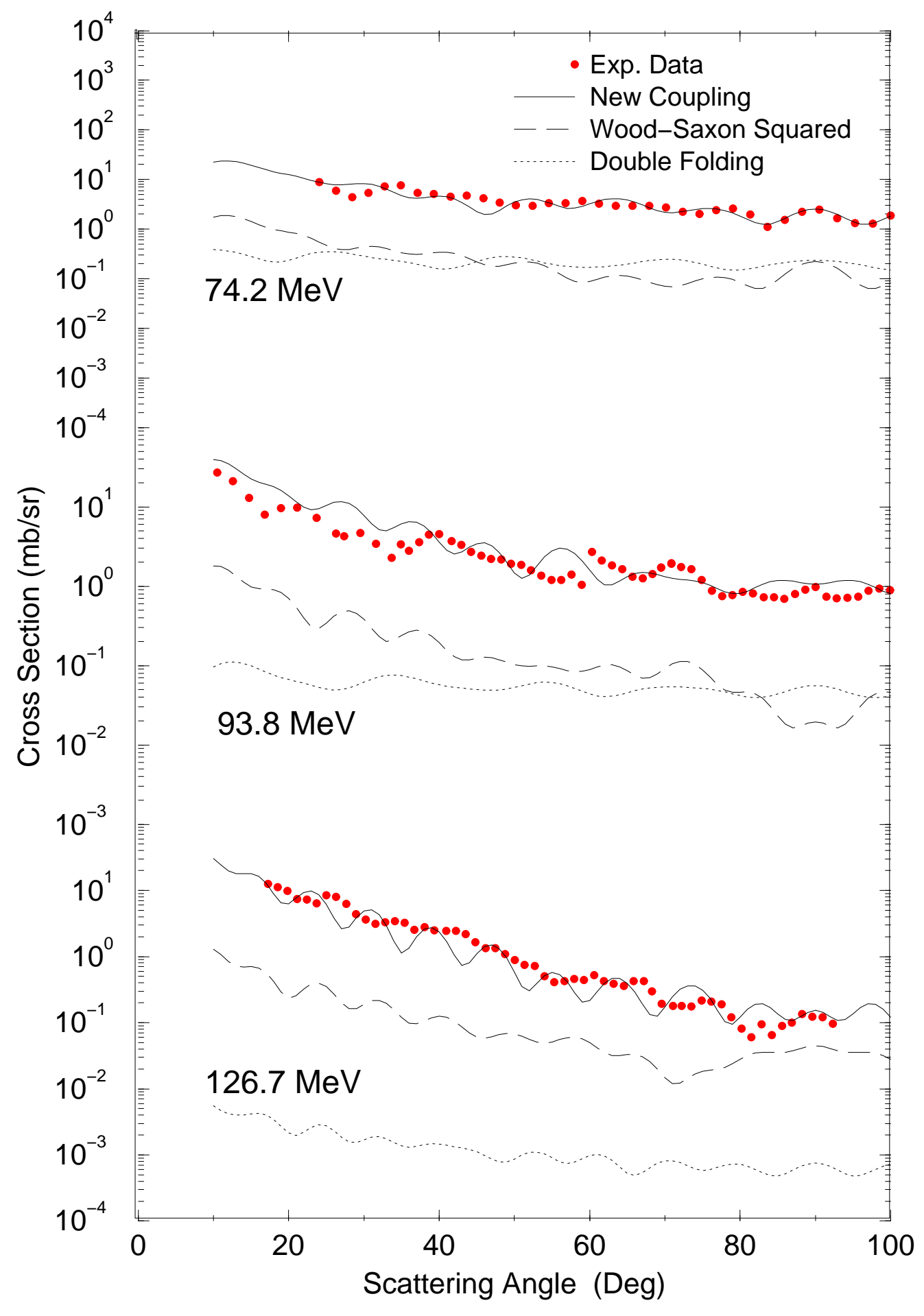

FIG. 4: Mutual-2 ${ }^{+}$state results. The legends are the same as figure 2, 